PROCEEDINGS OF THE

AMERICAN MATHEMATICAL SOCIETY

Volume 132, Number 9, Pages 2619-2628

S 0002-9939(04)07484-2

Article electronically published on April 21, 2004

\title{
A GEOMETRIC INEQUALITY AND A LOW $M$-ESTIMATE
}

\author{
BO'AZ KLARTAG
}

(Communicated by N. Tomczak-Jaegermann)

\begin{abstract}
We present an integral inequality connecting volumes and diameters of sections of a convex body. We apply this inequality to obtain some new inequalities concerning diameters of sections of convex bodies, among which is our "low $M$-estimate". Also, we give novel, alternative proofs to some known results, such as the fact that a finite volume ratio body has proportional sections that are isomorphic to a Euclidean ball.
\end{abstract}

\section{INTRODUCTION}

Let $K \subset \mathbb{R}^{n}$ be a convex body that contains the origin and has a nonempty interior. Denote $D_{n}=\left\{x \in \mathbb{R}^{n} ;|x| \leq 1\right\}$ the unit Euclidean ball in $\mathbb{R}^{n}$, where $|\cdot|$ is the standard Euclidean norm. We denote the volume-radius of $K \subset \mathbb{R}^{n}$ by

$$
v \cdot r a d .(K)=\left(\frac{\operatorname{Vol}(K)}{\operatorname{Vol}\left(D_{n}\right)}\right)^{1 / n} .
$$

The meaning of Vol here is clear: the standard Lebesgue measure in $\mathbb{R}^{n}$. However, in general, when we write $\operatorname{Vol}(K)$ or v.rad. $(K)$ for a body $K$ of smaller dimension, the quantity should be interpreted in the corresponding ambient subspace (i.e., the affine hull of $K)$. Denote also the diameter of $K$ as $\operatorname{diam}(K)=\sup _{x, y \in K}|x-y|$. A particular case of our inequality states that if $\operatorname{Vol}(K)=\operatorname{Vol}\left(D_{n}\right)$ and $n$ is even, then

$$
\int_{G_{n, n / 2}} \operatorname{v.rad} \cdot(K \cap E) \operatorname{diam}(K \cap E) d \mu(E)<c
$$

where $G_{n, n / 2}$ is the Grassmanian of $n / 2$-dimensional subspaces in $\mathbb{R}^{n}$, and $\mu$ is the unique rotation-invariant probability measure on $G_{n, n / 2}$. By $c, c^{\prime}, c_{1}, C$ etc. we denote numerical constants, whose value is not necessarily equal in various appearances. In addition, we obtain strong concentration of the integrand in (1.1):

$$
\mu\left\{E \in G_{n, n / 2} ; \operatorname{virad} .(K \cap E) \operatorname{diam}(K \cap E)>c_{1}\right\}<e^{-n} .
$$

The complete version of our inequality is presented in Proposition[1.1. This inequality has many interesting applications in asymptotic convex geometry, as demonstrated in Section 3, For instance, it suggests a "low $M$-estimate", which is dual in

Received by the editors May 26, 2003.

2000 Mathematics Subject Classification. Primary 46B20, 52A20, 52A40.

Key words and phrases. Asymptotic geometric analysis, diameters of sections.

This research was partially supported by the Israel Science Foundation and by the Minkowski Center for Geometry. 
some sense to Milman's low $M^{*}$-estimate ([M1], $\left.\left.\overline{\mathrm{PT}}\right], \mathrm{G0}\right)$, and provides a global proof of the statement due to Szarek/Tomczak-Jaegermann ( $[\mathrm{Sz}],[\mathrm{SzT}])$ that a finite volume ratio body has sections of proportional dimension that are isomorphic to a Euclidean ball.

Proposition 1.1. Let $K \subset \mathbb{R}^{n}$ be a convex body with a nonempty interior, that contains the origin, and let $1 \leq k \leq n$ be an integer. Denote $k=\lambda n$. Then,

$$
\int_{G_{n, k}} \operatorname{v.rad} .(K \cap E)^{\lambda} \operatorname{diam}(K \cap E)^{1-\lambda} d \mu(E)<C v . r a d .(K)
$$

where $\mu$ is the unique rotation-invariant probability measure on $G_{n, k}$ and $C>0$ is a numerical constant. Moreover,

$$
\mu\left\{E \in G_{n, k} ; \frac{v \cdot r a d .(K \cap E)^{\lambda} \operatorname{diam}(K \cap E)^{1-\lambda}}{v \cdot r a d .(K)}>C\right\}<e^{-n} .
$$

\section{Proof of the inequality}

The central component of the proof is the following simple formula, which follows from integration in polar coordinates.

Lemma 2.1. For any star-shaped $K \subset \mathbb{R}^{n}$ (i.e., $t K \subset K$ for $0 \leq t \leq 1$ ), and any integer $1 \leq k \leq n$,

$$
\int_{G_{n, k}} \int_{K \cap E}|x|^{n-k} d x d \mu(E)=\frac{k \operatorname{Vol}\left(D_{k}\right)}{n \operatorname{Vol}\left(D_{n}\right)} \operatorname{Vol}(K)
$$

where $\mu$ is the unique rotation-invariant probability measure on $G_{n, k}$, and the measure $d x$ is the natural Lebesgue measure in the appropriate subspace.

Proof. Denote $\kappa_{m}=\operatorname{Vol}\left(D_{m}\right)$. Let $\chi_{K}$ be the characteristic function of $K \subset \mathbb{R}^{n}$. Integrating in polar coordinates yields

$$
\begin{aligned}
& \int_{G_{n, k}} \int_{K \cap E}|x|^{n-k} d x d \mu(E) \\
& \quad=\int_{G_{n, k}} k \kappa_{k} \int_{S(E)} \int_{0}^{\infty} \chi_{K}(r \theta) r^{n-k} r^{k-1} d r d \sigma_{E}(\theta) d \mu(E)
\end{aligned}
$$

where $S(E)$ is the unit sphere in $E$ and $\sigma_{E}$ is the Haar probability measure on that sphere. Denote $S^{n-1}=S\left(\mathbb{R}^{n}\right)$ and $\sigma_{n}=\sigma_{\mathbb{R}^{n}}$. Now,

$$
\int_{G_{n, k}} \int_{S(E)} \int_{0}^{\infty} \chi_{K}(r \theta) r^{n-1} d r d \sigma_{E}(\theta) d \mu(E)=\int_{S^{n-1}} \int_{0}^{\infty} \chi_{K}(r \theta) r^{n-1} d r d \sigma_{n}(\theta)
$$

because of the rotation invariancy of both probability measures. With an additional application of polar integration we obtain

$$
k \kappa_{k} \int_{S^{n-1}} \int_{0}^{\infty} \chi_{K}(r \theta) r^{n-1} d r d \sigma_{n}(\theta)=\frac{k \kappa_{k}}{n \kappa_{n}} \int_{\mathbb{R}^{n}} \chi_{K}(x) d x=\frac{k \kappa_{k}}{n \kappa_{n}} \operatorname{Vol}(K),
$$

and the lemma is proved.

The following lemma is somewhat standard, following from simple convexity principles. Yet, for completeness we shall state it and prove it here. 
Lemma 2.2. Let $K \subset \mathbb{R}^{n}$ be a convex compact body that contains the origin, and let $r=\max _{x \in K}|x|$. Then,

$$
\operatorname{Vol}\left\{x \in K ;|x|>\frac{r}{2}\right\}>c^{n} \operatorname{Vol}(K)
$$

where $c>0$ is a numerical constant.

Proof. Clearly, we may assume that $\operatorname{Vol}(K)=1$. There exists $x_{0} \in K$ that satisfies $\left|x_{0}\right|=r$. Denote

$$
K_{t}=K \cap\left\{x \in \mathbb{R}^{n} ;\left\langle x, \frac{x_{0}}{\left|x_{0}\right|}\right\rangle=t\right\}, \quad \varphi(t)=\operatorname{Vol}_{n-1}\left(K_{t}\right) .
$$

By maximality of $\left|x_{0}\right|$, the support of $\varphi$ is contained in $[-r, r]$ and $\int_{-r}^{r} \varphi=1$. It is sufficient to prove that

$$
\int_{r / 2}^{r} \varphi>c^{n}
$$

since in that case,

$$
\operatorname{Vol}\left\{x \in K ;|x|>\frac{r}{2}\right\} \geq \operatorname{Vol}\left\{x \in K ;\left\langle x, \frac{x_{0}}{\left|x_{0}\right|}\right\rangle>\frac{r}{2}\right\}>c^{n} .
$$

Let us prove inequality (2.1). Denote $M=\sup \varphi$, and let $-r \leq y_{0} \leq r$ be a point such that $\varphi\left(y_{0}\right)>\frac{M}{2}$. Let $\frac{1}{2} r \leq x \leq \frac{3}{4} r$ be an arbitrary point. Then either $x \in\left[y_{0}, r\right]$ or $x \in\left[0, y_{0}\right]$. In the first case, by convexity,

$$
\frac{r-x}{r-y_{0}} K_{y_{0}}+\frac{x-y_{0}}{r-y_{0}} x_{0} \subset K_{x},
$$

and

$$
\varphi(x)=\operatorname{Vol}_{n-1}\left(K_{x}\right) \geq\left(\frac{r-x}{r-y_{0}}\right)^{n-1} \operatorname{Vol}_{n-1}\left(K_{y_{0}}\right) \geq \frac{1}{8^{n-1}} \varphi\left(y_{0}\right)=\frac{M}{2 \cdot 8^{n-1}}
$$

since $r-x \geq \frac{r}{4}$ and $r-y_{0} \leq 2 r$. In the second case, $\frac{x}{y_{0}} K_{y_{0}} \subset K_{x}$ since $0 \in K$, and

$$
\varphi(x) \geq\left(\frac{x}{y_{0}}\right)^{n-1} \varphi\left(y_{0}\right) \geq \frac{M}{2^{n}} .
$$

To conclude, for any $\frac{1}{2} r \leq x \leq \frac{3}{4} r$ we have $\varphi(x) \geq \frac{M}{8^{n}}$ and

$$
\int_{r / 2}^{r} \varphi(x) d x>\int_{\frac{1}{2} r}^{\frac{3}{4} r} \varphi(x) d x \geq \frac{M}{8^{n}} \frac{r}{4} \geq \frac{1}{2 \cdot 8^{n}}
$$

since $1=\int_{-r}^{r} \varphi \leq 2 r M$, and (2.1) is proved.

Proof of Proposition 1.1. By Lemma 2.1,

$$
\left(\int_{G_{n, \lambda n}} \int_{K \cap E}|x|^{(1-\lambda) n} d x d \mu(E)\right)^{1 / n}=\left(\frac{\lambda n \kappa_{\lambda n}}{n \kappa_{n}} \operatorname{Vol}(K)\right)^{1 / n}
$$

where again we use the notation $\kappa_{m}=\operatorname{Vol}\left(D_{m}\right)$. Regarding the left-hand side of (2.2), from Lemma 2.2,

$$
\begin{aligned}
c^{\lambda n} \operatorname{Vol}(K \cap E)\left(\frac{\operatorname{diam}(K \cap E)}{4}\right)^{(1-\lambda) n} & \leq \int_{K \cap E}|x|^{(1-\lambda) n} d x \\
& \leq \operatorname{Vol}(K \cap E) \operatorname{diam}(K \cap E)^{(1-\lambda) n}
\end{aligned}
$$


since

$$
\frac{\operatorname{diam}(K \cap E)}{2} \leq \max _{x \in K \cap E}|x| \leq \operatorname{diam}(K \cap E) .
$$

Let us introduce a simplifying notation: when we write $A \approx B$, we mean that there exist two numerical constants $c_{1}, c_{2}>0$ such that $c_{1} A<B<c_{2} A$. Hence,

$$
\begin{aligned}
& \left(\int_{G_{n, \lambda n}} \int_{K \cap E}|x|^{(1-\lambda) n} d x d \mu(E)\right)^{1 / n} \\
& \quad \approx\left(\int_{G_{n, \lambda n}} \operatorname{Vol}(K \cap E) \operatorname{diam}(K \cap E)^{(1-\lambda) n} d \mu(E)\right)^{1 / n} .
\end{aligned}
$$

Combining (2.2) and (2.3), and using $\operatorname{Vol}(K)=\operatorname{vrrad}(K)^{n} \kappa_{n}, \operatorname{Vol}(K \cap E)=$ v.rad. $(K \cap E)^{\lambda n} \kappa_{\lambda n}$ and $\lambda^{1 / n} \approx 1$ (since $\frac{1}{n} \leq \lambda \leq 1$ ), we obtain

$$
\left(\int_{G_{n, \lambda n}} \operatorname{verad} .(K \cap E)^{\lambda n} \operatorname{diam}(K \cap E)^{(1-\lambda) n} d \mu(E)\right)^{1 / n} \approx \operatorname{verad} .(K) .
$$

Using Markov's inequality $\operatorname{Prob}\left\{f>t\|f\|_{p}\right\} \leq t^{-p}$ for $p=n$ and $t=e$, yields

$$
\mu\left\{E \in G_{n, k} ; \text { v.rad. }(K \cap E)^{\lambda} \operatorname{diam}(K \cap E)^{1-\lambda}>e C v \cdot r a d .(K)\right\}<e^{-n},
$$

which proves (1.3). Obtaining (1.2) is now easy. By Jensen's inequality,

$$
\int_{G_{n, \lambda n}} \operatorname{vrrad} .(K \cap E)^{\lambda} \operatorname{diam}(K \cap E)^{1-\lambda} d \mu(E)<C^{\prime} \operatorname{vrad} .(K) .
$$

Remark 2.3. Lemma 2.2 uses the convexity of the body $K$. Nevertheless, weaker notions than convexity are sufficient for obtaining the conclusion of this lemma. For example, quasiconvexity is enough. Since the convexity assumption is used only in the proof of Lemma 2.2 the conclusions of Proposition 1.1 hold for quasiconvex bodies as well.

\section{Corollaries of the inequality}

A few corollaries of Proposition 1.1 are presented in this section. Some of these are known, yet our proof is completely different from their usual proofs, and some are new, like the "low $M$-estimate" for diameters of proportional sections, and others.

\subsection{The volume of proportional sections is not large.}

Corollary 3.1. Let $K \subset \mathbb{R}^{n}$ be a convex body that contains the origin and has a nonempty interior, and let $1 \leq k \leq n$ be an integer. Then a random subspace $E \in G_{n, k}$ satisfies

$$
\text { v.rad. }(K \cap E)<C v \text { rad. }(K)
$$

with probability greater than $1-e^{-n}$, where $C$ is a numerical constant, independent of $k$ and $n$. 
Proof. Clearly v.rad. $(K \cap E) \leq \frac{1}{2} \operatorname{diam}(K \cap E)$ by the isodiametric inequality in $\mathbb{R}^{n}$ (see $[\overline{\mathrm{BF}}$, page 77 ). Therefore, (1.3) transforms to

$$
\mu\left\{E \in G_{n, k} ; \operatorname{vrad} .(K \cap E)>C v \cdot r a d .(K)\right\}<e^{-n} .
$$

\subsection{Diameters of sections via orthogonal projection.}

Lemma 3.2. Let $K \subset \mathbb{R}^{n}$ be a convex body such that the origin is its barycenter. Let $E$ be any subspace of dimension $k=\lambda n$. Then,

$$
\operatorname{verad} .(K \cap E)>c(\lambda) \frac{v \cdot r a d .(K)^{\frac{1}{\lambda}}}{v \cdot r a d .\left(\operatorname{Proj}_{E^{\perp}}(K)\right)^{\frac{1-\lambda}{\lambda}}}
$$

where $\operatorname{Proj}_{E} \perp$ is the orthogonal projection onto $E^{\perp}$, and $c(\lambda) \approx C \sqrt{\lambda}$.

Proof. By $[\mathrm{Sp}$,

$$
\operatorname{Vol}(K) \leq \operatorname{Vol}\left(\operatorname{Proj}_{E^{\perp}}(K)\right) \operatorname{Vol}(K \cap E)
$$

and, raising to the power of $\frac{1}{\lambda n}$, we obtain

$$
\operatorname{verad} .(K \cap E) \geq\left(\frac{\kappa_{n}}{\kappa_{\lambda n} \kappa_{(1-\lambda) n}}\right)^{\frac{1}{\lambda n}} \frac{\operatorname{vrrad} .(K)^{\frac{1}{\lambda}}}{\operatorname{vrrad} .\left(\operatorname{Proj}_{E^{\perp}}(K)\right)^{\frac{1-\lambda}{\lambda}}} .
$$

Since $\sqrt{m} \kappa_{m}^{1 / m} \approx 1$, we have $\left(\frac{\kappa_{n}}{\kappa_{\lambda n} \kappa_{(1-\lambda) n}}\right)^{1 / n} \approx \lambda^{\frac{\lambda}{2}}(1-\lambda)^{\frac{1-\lambda}{2}} \approx 1$ and the lemma is proved.

Corollary 3.3. Let $K \subset \mathbb{R}^{n}$ be a convex body such that the origin is its barycenter. Let $1 \leq k \leq n$ be an integer, $k=\lambda n$. Then a random subspace $E \in G_{n, k}$ satisfies

$$
\operatorname{diam}(K \cap E)<c(\lambda) v \cdot r a d .\left(\operatorname{Proj}_{E^{\perp}}(K)\right)
$$

with probability greater than $1-e^{-n}$, where $\operatorname{Proj}_{E^{\perp}}$ is the orthogonal projection onto $E^{\perp}$, and $c(\lambda) \approx C^{\frac{1}{1-\lambda}}$.

Proof. By Lemma 3.2

$$
\frac{v \cdot r a d .(K)^{\frac{1}{1-\lambda}}}{v \cdot r a d .(K \cap E)^{\frac{\lambda}{1-\lambda}}}<C v \cdot r a d .\left(\operatorname{Proj}_{E^{\perp}}(K)\right)
$$

and by Proposition 1.1, with probability greater than $1-e^{-n}$,

$$
\operatorname{diam}(K \cap E)<\tilde{C}^{\frac{1}{1-\lambda}} \frac{v \cdot r a d .(K)^{\frac{1}{1-\lambda}}}{v \cdot r a d .(K \cap E)^{\frac{\lambda}{1-\lambda}}}<c(\lambda) \operatorname{vrad} \cdot\left(\operatorname{Proj}_{E^{\perp}}(K)\right)
$$

where $c(\lambda)=C \cdot \tilde{C}^{\frac{1}{1-\lambda}}$.

Remark 3.4. Note that the low $M^{*}$-estimate (Theorem 3.8 here) follows from Corollary 3.3 with a poorer dependence on $\lambda$. This follows from

$$
v \cdot \operatorname{rad} .\left(\operatorname{Proj}_{E}{ }(K)\right) \leq M^{*}\left(\operatorname{Proj}_{E \perp}(K)\right) \leq c \sqrt{\frac{1}{1-\lambda}} M^{*}(K)
$$

by Urysohn's inequality and a contraction principle (GM3], Section 4.2). 
3.3. Euclidean sections of finite volume ratio bodies. Here we provide another proof for the fact discovered by Szarek and Tomczak-Jaegermann ( $[\mathrm{Sz}],[\mathrm{SzT}])$, regarding sections of bodies with a finite volume ratio. The common proof of this fact uses some concentration inequalities and an argument involving nets. Let us give an alternative proof, based on Proposition 1.1 which is a "global" one and avoids the use of nets (see also $[\mathrm{B}]$ and $[\mathrm{K}]$ ).

Corollary 3.5. Let $K \subset \mathbb{R}^{n}$ be a convex body, such that $D_{n} \subset K$ and such that $v$.rad. $(K)<\alpha$. Let $1 \leq k \leq n$ be an integer, $k=\lambda n$. Then a random subspace $E \in G_{n, k}$ satisfies

$$
D_{k} \subset K \cap E \subset c(\lambda, \alpha) D_{k}
$$

with probability greater than $1-e^{-n}$, where $c(\lambda, \alpha) \approx(C \alpha)^{\frac{1}{1-\lambda}}$.

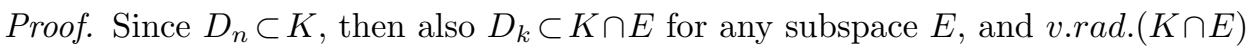
$\geq 1$. It is enough to bound the diameter of a random section. According to (1.3),

$$
\begin{aligned}
& \mu\left\{E \in G_{n, k} ; \operatorname{diam}(K \cap E)>(C \alpha)^{\frac{1}{1-\lambda}}\right\} \\
& \leq \mu\left\{E \in G_{n, k} ; \frac{\text { v.rad. }(K \cap E)^{\lambda} \operatorname{diam}(K \cap E)^{1-\lambda}}{\text { v.rad. }(K)}>C\right\}<e^{-n}
\end{aligned}
$$

and the corollary is proved. Note that we get $c(\lambda, \alpha)<(C \alpha)^{\frac{1}{1-\lambda}}$, which is the same dependence obtained in $[\mathrm{Sz}]$ and $[\mathrm{SzT}]$.

3.4. Bounded sections in $M$-position. Let $K \subset \mathbb{R}^{n}$ be a convex body, such that $\operatorname{Vol}(K)=\operatorname{Vol}\left(D_{n}\right)$ and such that the covering number

$$
N\left(K, D_{n}\right)=\inf \left\{\sharp A ; K \subset A+D_{n}\right\}<e^{c n}
$$

for some constant $c>0$, where $\sharp A$ is the number of elements in the set $A$. Under these conditions, we say that $K$ is in $M$-position with constant $c$ (see [M3], M4] or $[\mathrm{P}]$, chapter 7 , for different proofs of the fact that any convex body has a linear image that is in $M$-position with some absolute constant). Our next corollary provides a simple proof for a result in the spirit of [GM1], section 2.3 and [GM2], section 5 .

Corollary 3.6. Let $K \subset \mathbb{R}^{n}$ be a convex body in $M$-position, such that the origin is its barycenter, and such that $\operatorname{Vol}(K)=\operatorname{Vol}\left(D_{n}\right)$. Let $1 \leq k \leq n$ be an integer, $k=\lambda n$. Then a random subspace $E \in G_{n, k}$ satisfies

$$
c_{1}(\lambda)<\operatorname{diam}(K \cap E)<c_{2}(\lambda)
$$

with probability greater than $1-e^{-n}$, where $c_{1}(\lambda) \approx c^{\frac{1}{\lambda}}$ and $c_{2}(\lambda) \approx C^{\frac{1}{1-\lambda}}$.

Proof. Begin by estimating volumes of projections. From (3.1),

$$
\text { v.rad. }\left(\operatorname{Proj}_{E^{\perp}}(K)\right) \leq N\left(K, D_{n}\right)^{\frac{1}{(1-\lambda) n}} \operatorname{vrad} .\left(\operatorname{Proj}_{E^{\perp}}\left(D_{n}\right)\right)<e^{\frac{c}{1-\lambda}} .
$$

By Lemma 3.2

$$
v \cdot \operatorname{rad} .(K \cap E)>c(\lambda) \frac{1}{v \cdot \operatorname{rad} .\left(\operatorname{Proj}_{E^{\perp}}(K)\right)^{\frac{1-\lambda}{\lambda}}}>c^{\frac{1}{\lambda}} .
$$


This is true for any subspace $E \in G_{n, k}$. Hence, for any subspace $E$ of dimension $k$, we have $\operatorname{diam}(K \cap E) \geq 2 v \operatorname{rad} .(K \cap E)>c_{1}(\lambda)$. We continue as in the proof of Corollary 3.5 :

$$
\begin{aligned}
\mu & \left\{E \in G_{n, k} ; \operatorname{diam}(K \cap E)>c_{2}(\lambda)\right\} \\
& \leq \mu\left\{E \in G_{n, k} ; \frac{v \cdot \operatorname{rad} .(K \cap E)^{\lambda} \operatorname{diam}(K \cap E)^{1-\lambda}}{\operatorname{vrad} .(K)}>C\right\}<e^{-n} .
\end{aligned}
$$

Remark 3.7. The right-most inequality in (3.2) also follows from Corollary 3.5 by considering the body $K+D_{n}$, and observing that v.rad. $\left(K+D_{n}\right)<c$ when $K$ is in $M$-position.

3.5. Low $M$-estimate. Here we prove a dual estimate to the well-known low $M^{*}$ estimate (Theorem 3.8 here), which involves the "dual parameter" $M$ of the body. Let us define these parameters. Let $K \subset \mathbb{R}^{n}$ be a convex body such that the origin is in its interior. Denote the gauge function of $K$ as

$$
\|x\|=\inf \{\lambda>0 ; x \in \lambda K\} .
$$

Note that the gauge function is homogeneous in $\mathbb{R}^{n}$ and satisfies the triangle inequality. However, it is not symmetric (not necessarily $\|x\|=\|-x\|$ ); hence it may not be a norm. Define

$$
M(K)=\int_{S^{n-1}}\|x\| d \sigma(x)
$$

where $\sigma$ is the unique rotation-invariant probability measure on $S^{n-1}$. The polar body of $K$ is $K^{\circ}=\left\{x \in \mathbb{R}^{n} ; \forall y \in K,\langle x, y\rangle \leq 1\right\}$, and we write $M^{*}(K)=M\left(K^{\circ}\right)$. The low $M^{*}$-estimate is the following theorem (see [M1], [PT], or [Go] for best dependence on $\lambda$ ).

Theorem 3.8 (Low $M^{*}$-estimate). Let $K \subset \mathbb{R}^{n}$ be a convex body with the origin in its interior. Let $1 \leq k \leq n$ be an integer, $k=\lambda n$. Then a random subspace $E \in G_{n, k}$ satisfies

$$
\begin{gathered}
\qquad \operatorname{diam}(K \cap E)<c(\lambda) M^{*}(K) \\
\text { with probability greater than } 1-e^{-\tilde{c} \cdot(1-\lambda) n}, \text { where } c(\lambda) \approx \frac{1}{\sqrt{1-\lambda}} .
\end{gathered}
$$

The low $M^{*}$-estimate is a fundamental inequality in the study of high-dimensional convex bodies. It is crucially involved, for example, in the proof of Milman's quotient of subspace theorem M2. A dual form of the low $M^{*}$-estimate appears in [GM1], under the name "conditional low $M$-estimate", since it requires that $M(K)$ be very close to $\sup _{x \in S^{n-1}}\|x\|$. Here we present a different estimate, which is valid for all levels of $M(K)$.

Proposition 3.9 (Low $M$-estimate). Let $K \subset \mathbb{R}^{n}$ be a convex body with the origin in its interior. Let $1 \leq k \leq n$ be an integer, $k=\lambda n$. Then a random subspace $E \in G_{n, k}$ satisfies

$$
\operatorname{diam}(K \cap E)<c(\lambda) M(K)^{\frac{\lambda}{1-\lambda}} \operatorname{vrad} \cdot(K)^{\frac{1}{1-\lambda}}
$$

with probability greater than $1-e^{-n}$, where $c(\lambda) \approx C^{\frac{1}{1-\lambda}}$. 
Proof. By Proposition 1.1 a random section satisfies

$$
\operatorname{diam}(K \cap E)^{1-\lambda}<C \frac{\text { v.rad. }(K)}{\operatorname{v.rad.}(K \cap E)^{\lambda}}
$$

with the desired probability. According to Jensen's inequality,

$$
M(K \cap E)=\int_{S(E)}\|x\| d \sigma_{E}(x) \geq\left(\int_{S(E)}\|x\|^{-k} d \sigma_{E}(x)\right)^{-1 / k}=\frac{1}{v \cdot \operatorname{rad} .(K \cap E)}
$$

and, by substituting into (3.3), we get

$$
\operatorname{diam}(K \cap E)^{1-\lambda}<C M(K \cap E)^{\lambda} \text { v.rad. }(K) .
$$

By a contraction principle, for any subspace $E$ we have $M(K \cap E)<c \sqrt{\frac{1}{\lambda}} M(K)$ (see e.g. GM3], Section 4.2). Hence, with the appropriate probability,

$$
\operatorname{diam}(K \cap E)^{1-\lambda}<c C \lambda^{-\frac{\lambda}{2}} M(K)^{\lambda} v \cdot r a d .(K)<C^{\prime} M(K)^{\lambda} v . r a d .(K)
$$

and raising to the power of $\frac{1}{1-\lambda}$ obtains the proposition.

Remark 3.10. By imitating the technique of [GM1], GM2], one can use the low $M$-estimate to show that if $R>0$ satisfies

$$
M\left(K \cap R D_{n}\right)^{\frac{\lambda}{1-\lambda}} v \cdot r a d .\left(K \cap R D_{n}\right)^{\frac{1}{1-\lambda}} \approx c(\lambda) R,
$$

then for a random $\lambda n$-dimensional subspace $E$, we have $\operatorname{diam}(K \cap E) \leq R$.

3.6. Distances to Euclidean ball. Given a convex body $K \subset \mathbb{R}^{n}$ that contains the origin in its interior, we introduce the outer and inner radius of $K$, respectively:

$$
a(K)=\inf \left\{a>0 ; K \subset a D_{n}\right\}, b(K)=\inf \left\{b>0 ; \frac{1}{b} D_{n} \subset K\right\} .
$$

Note that $a(K) \approx \operatorname{diam}(K)$ and $b(K) \approx \operatorname{diam}\left(K^{\circ}\right)$. The "geometric distance" of $K$ to a Euclidean ball is simply $d\left(K, D_{n}\right)=a(K) b(K)$. This can be written as follows:

$$
d\left(K, D_{n}\right)=\frac{a(K)}{v \cdot r a d \cdot(K)} \frac{v \cdot r a d \cdot(K)}{1 / b(K)} .
$$

Note that both terms are not smaller than one. The first term measures to what extent the body "fills" the circumscribing Euclidean ball, while the second term measures how much volume of $K$ is captured by the circumscribed Euclidean ball. We prove the following corollary, just to indicate a possible application of the combination of the low $M^{*}$-estimate with our low $M$-estimate.

Corollary 3.11. Let $K \subset \mathbb{R}^{n}$ be a convex body that contains the origin in its interior and such that $\operatorname{Vol}(K)=\operatorname{Vol}\left(D_{n}\right)$. Let $E \subset \mathbb{R}^{n}$ be a random subspace of dimension $\left\lfloor\frac{n}{2}\right\rfloor$. Then,

$$
K \cap E \subset c \min \left\{M(K), M^{*}(K)\right\} D_{n} \subset c^{\prime} \sqrt{d(K, D)} D_{n}
$$

with probability greater than $1-e^{-n}$, where $c, c^{\prime}>0$ are numerical constants.

Proof. According to Corollary 3.9, a random $\left\lfloor\frac{n}{2}\right\rfloor$-dimensional subspace $E \subset \mathbb{R}^{n}$ satisfies

$$
K \cap E \subset c M(K) D_{n} .
$$

Also, according to Theorem 3.8

$$
K \cap E \subset c^{\prime} M^{*}(K) D_{n}
$$


with high probability. Hence, a random section of dimension $\lfloor n / 2\rfloor$ satisfies

$$
K \cap E \subset C \min \left\{M(K), M^{*}(K)\right\} D_{n} \subset C^{\prime} \sqrt{M(K) M^{*}(K)} D_{n}
$$

and since $M(K) \leq b(K), M^{*}(K) \leq a(K)$, we get that for a random section,

$$
K \cap E \subset \tilde{c} \sqrt{d(K, D)} D_{n} .
$$

3.7. Diameters of low-dimensional sections. We will show that given a "nondegenerate" arbitrary convex body $K \subset \mathbb{R}^{n}$, its sections of small dimension (up to $\frac{n}{\log n}$, almost proportional sections) typically have a small diameter, i.e., with high probability their diameter is not much greater than the volume radius of the body. By saying "non-degenerate" we mean that $K$ is not essentially a body of smaller dimension. For example, it should contain some small $n$-dimensional ball.

Corollary 3.12. Let $\alpha>0$, and assume that $n>n(\alpha)=e^{2 \alpha}$ is an integer. Let $K \subset \mathbb{R}^{n}$ be a convex body that contains the origin, and such that $\operatorname{Vol}(K)=\operatorname{Vol}\left(D_{n}\right)$. Assume also that $\frac{1}{n^{t}} D_{n} \subset K$ for some $t>0$. Let $1 \leq k \leq \alpha \frac{n}{\log n}$ be an integer. Then, a random subspace $E \in G_{n, k}$ satisfies

$$
\operatorname{diam}(K \cap E)<c(\alpha, t)
$$

with probability greater than $1-e^{-n}$, where $c(\alpha, t)=C^{t \alpha}$.

Proof. Since $n>n(\alpha)$, we know that $\alpha<\frac{\log n}{2}$. By Proposition 1.1 a random subspace $E \in G_{n, k}$ satisfies

$$
\operatorname{diam}(K \cap E)<\frac{c}{\text { v.rad. }(K \cap E)^{\frac{k}{n-k}}}<\frac{c}{\text { v.rad. }(K \cap E)^{\frac{2 \alpha}{\log n}}}
$$

with probability greater than $1-e^{-n}$. But since $\frac{1}{n^{t}} D_{n} \subset K$, we get that

$$
\text { v.rad. }(K \cap E) \geq \frac{1}{n^{t}} .
$$

Hence, for a random subspace $E \in G_{n, k}$,

$$
\operatorname{diam}(K \cap E)<c n^{\frac{2 t \alpha}{\log n}}=c e^{2 t \alpha} .
$$

\section{ACKNOWLEDGMENT}

I would to thank V. Milman for encouraging me to write this paper and for his valuable suggestions during the research. Thanks also to M. Fradelizi for referring me to the paper $[\mathrm{Sp}$.

\section{REFERENCES}

[B] K. M. Ball, An Elementary Introduction to Modern Convex Geometry, appeared in Flavors of Geometry, Ed. S. Levy, Mathematical Sciences Research Institute Publications, vol. 31, Cambridge University Press, Cambridge, 1997. MR 98g:00024

[BF] T. Bonnesen and W. Fenchel, Theorie der konvexen Körper, Springer-Verlag, Berlin, 1934; English transl. Theory of convex bodies, BCS Associates, Moscow, 1987. MR 88j:52001

[GM1] A. A. Giannopoulos and V. D. Milman, On the diameter of proportional sections of a symmetric convex body, International Math. Research Notices, No. 1 (1997) 5-19. MR 97k:52003

[GM2] A. A. Giannopoulos and V. D. Milman, Mean width and diameter of proportional sections of a symmetric convex body, J. reine angew. Math. 497 (1998) 113-139. MR 99c:52006 
[GM3] A. A. Giannopoulos and V. D. Milman, Euclidean structure in finite dimensional normed spaces, Handbook of the Geometry of Banach spaces, W. B. Johnson, J. Lindenstrauss, eds., Elsevier Science, Vol. 1 (2001) 707-779. MR 2003b:46008

[Go] Y. Gordon, On Milman's inequality and random subspaces which escape through a mesh in $\mathbb{R}^{n}$, Geometric aspects of functional analysis (1986/87), Israel seminar, Lecture Notes in Mathematics, no. 1317, Springer-Verlag, Berlin, 1988, pp. 84-106. MR 89b:46004

[K] G. Kuperberg, Another low-technology estimate in convex geometry. Convex Geometric Analysis (Berkeley, CA, 1996), Math. Sci. Res. Inst. Publ. 34, Cambridge Univ. Press, (1999), 117-127. MR 99k:52006

[M1] V. D. Milman, Random subspaces of proportional dimension of finite-dimensional normed spaces: approach through the isoperimetric inequality, Banach spaces (Columbia, Mo., 1984), Lecture Notes in Mathematics, no. 1166, Springer-Verlag, Berlin, 1985, pp. 106115. MR 87j:46037b

[M2] V. D. Milman, Almost Euclidean quotient spaces of subspaces of a finite-dimensional normed space, Proc. Amer. Math. Soc. 94 (1985) 445-449. MR 86g:46025

[M3] V. D. Milman, Inégalité de Brunn-Minkowski inverse et applications à la théorie locale des espaces normés, C. R. Acad. Sci. Paris Sér. I Math. 302 (1986) 25-28. MR 87f:52018

[M4] V. D. Milman, Isomorphic symmetrizations and geometric inequalities, Geometric aspects of functional analysis (1986/87), Israel seminar, Lecture Notes in Mathematics, no. 1317, Springer-Verlag, Berlin, 1988, pp. 107-131. MR 89h:52011

[PT] A. Pajor and N. Tomczak-Jaegermann, Subspaces of small codimension of finitedimensional Banach spaces, Proc. Amer. Math. Soc. 97 (1986) 637-642. MR 87i:46040

[P] G. Pisier, The volume of convex bodies and Banach space geometry, Cambridge Tracts in Mathematics, vol. 94, Cambridge University Press, Cambridge, 1997. MR 91d:52005

[Sch] R. Schneider, Convex bodies: the Brunn-Minkowski theory, Encyclopedia of Mathematics and its Applications, 44, Cambridge University Press, Cambridge, 1993. MR 94d:52007

[Sp] J. E. Spingorn, An inequality for sections and projections of a convex set, Proc. Amer. Math. Soc. 118, no. 4 (1993), 1219-1224. MR 93j:52011

[Sz] S. Szarek, On Kashin's almost Euclidean orthogonal decomposition of $l_{n}^{1}$, Bull. Acad. Polon. Sci. Sér. Sci. Math. Astronom. Phys. 26, no. 8 (1978) 691-694. MR 80i:46020

[SzT] S. Szarek and N. Tomczak-Jaegermann, On nearly Euclidean decomposition for some classes of Banach spaces, Compositio Math. 40, no. 3, (1980) 367-385. MR 82e:46032

School of Mathematical Sciences, Tel-Aviv University, Tel-Aviv 69978, Israed

E-mail address: klartagb@post.tau.ac.il 